\title{
EXPERIMENTAL EVALUATION THE EFFECTIVENESS OF WATER MIST FIRE EXTINGUISHING SYSTEMS AT OIL AND GAS INDUSTRY
}

\author{
Nyashina G.S. ${ }^{1,}$ a Medvedev V.V. ${ }^{1}$, Shevyrev S.A. ${ }^{1}$, Vysokomornaya O.V. ${ }^{1}$ \\ ${ }^{1}$ National Research Tomsk Polytechnic University, 634050 Tomsk, Russia
}

\begin{abstract}
Currently mist water is one of the most promising areas of fire protection. We performed an experimental study of phase transformations drops of water mist (range 50 - 500 microns) in motion in a high-temperature (500 - $2000 \mathrm{~K}$ ) typical products of combustion of petroleum products (gasoline, kerosene, acetone, alcohol). We used high speed (the speed of shooting at least $10^{5}$ frames per second) and optical methods of recording streams of liquid and gas medium. We determined the effect of the parameters of the test process (the initial temperature and the initial droplet size) at the rate of evaporation of atomized water under these conditions.
\end{abstract}

\section{Introduction}

Objects of oil and gas industry are an important part of the fuel and energy complex of Russia The country has an extensive network of main oil pipelines, oil pipelines and gas pipelines. It passes through the territory of the majority of the Russian Federation subjects. Danger explosive situations constitute a large amount of combustible liquids under pressure on objects of the oil and gas industry. Liquids can spill on areas and burn over large areas at infringement of equipment. For a special danger in this case are oil products, which are located high in the areas near rivers, reservoirs, railways, roads and other communications. One of the ways that provide the prevention of emergencies is to equip the production facilities with modern fire protection systems [1].

Currently the installation of foam extinguishing is the main method of fire extinguishing at oil facilities. Foam is one of the most universal and effective substances for fires extinguishing in industrial plants, warehouses, drain-loading racks in the storage tanks, tank farms. Foam compositions are dispersions of gas bubbles surrounded by a liquid film. Foam has relatively aggregate and thermodynamic instability. However, the main disadvantage of foam systems is that the composition must be stirred continuously. In other foam exfoliate (foam and water separation) and lose quenching properties. Also, the foam of different chemical composition are not always harmless, and its drain into drains is not possible. Waste must be disposed after the fire. These disadvantages foams complicate its widespread and ubiquitous use [2].

Another popular method of fire protection is using system, which use water mist flow. Demand for water mist increases because of the water availability, its environmental cleanliness, safety and high extinguishing efficiency [3]. Water mist systems are used in facilities, where high effectiveness of extinguishing is required, water supply is limited and need to minimum damage of water use is required.

Water mist is considered water spray droplets with an average diameter of 150-500 microns, according to SFS 88-2001*. Special nozzles are used for formation water mist [4]. The water jet is passed through the nozzle under high pressure and crushed into droplets. Deluge and sprinkler automatic fire extinguishing, modular installation extinguishing fires, fire trunks, water mist fire extinguishers and water for fire fighting streams sprayed water jets are used to extinguish fires. [5]

Water mist cools shields, heat radiation and reduces the oxygen concentration. Water has a high specific heat of vaporization$2256 \mathrm{KJ} / \mathrm{kg}$, when it boils occurs dissipation of heat from the combustion zone. This effect can result in a complete stop of the combustion reaction. The steam formed in the combustion zone by evaporation of water.

Steam prevents gas exchange of the combustion products and oxygen, and also reduces the oxygen concentration near the combustion zone. Thus, the water, in addition to the cooling, implements two mechanism extinguishing insulation and dilution [6].

The purpose of this work is the experimental study of phase transformations of water droplets in their motion in gases by using modern methods of digital optical "tracer" visualization and high-speed video recording.

\section{Experimental setup and procedure}

\footnotetext{
${ }^{\mathrm{a} C}$ Corresponding author: gsn1@tpu.ru
} 


\section{EPJ Web of Conferences}

In experiments with water mist flows used liquid petroleum products such as gasoline, kerosene, acetone, technical, industrial alcohol, as well as oil of various grades.

All studies were performed using the experimental setup, a schematic diagram is shown in figure 1.

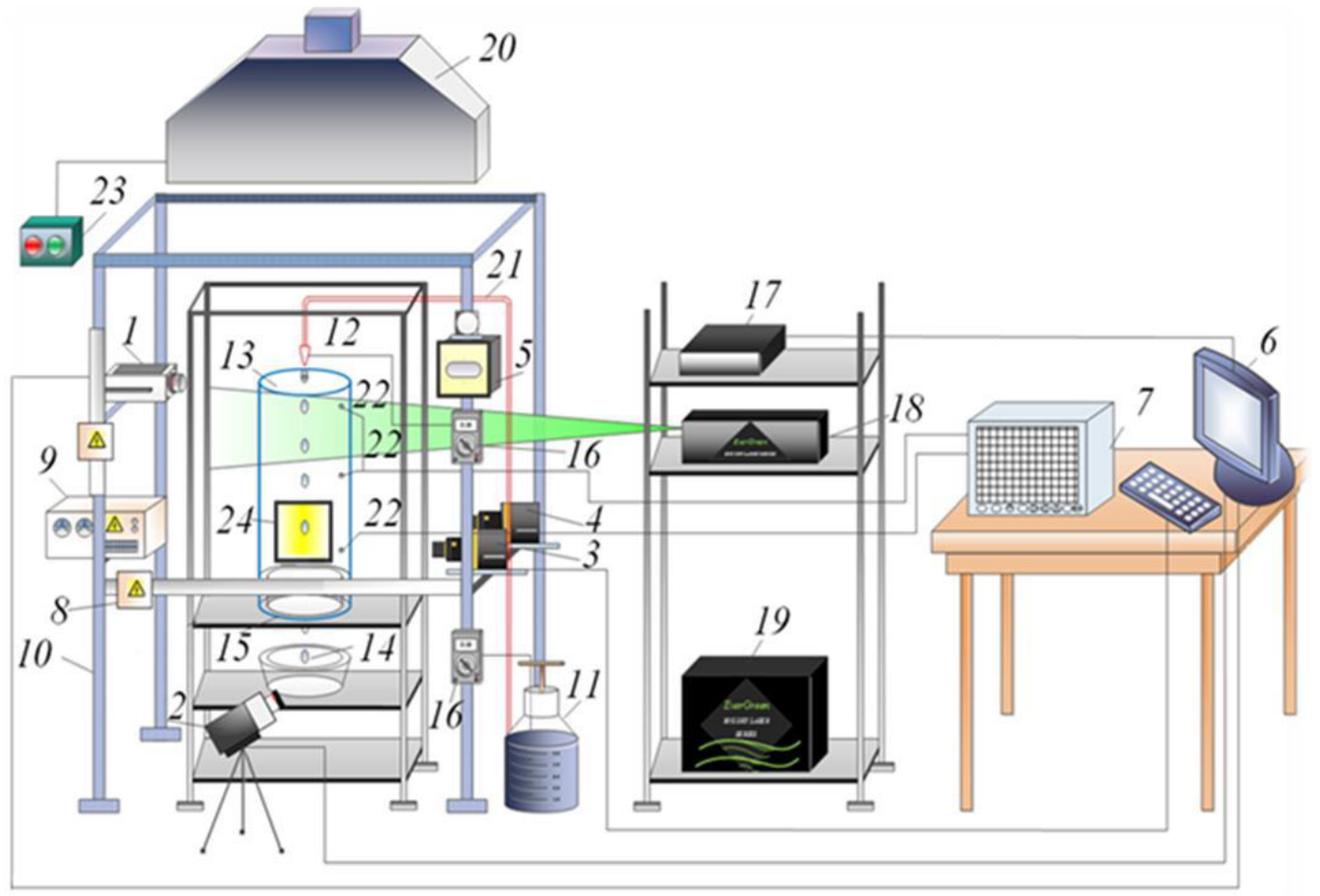

Figure 1. Scheme of an experimental setup: 1,2 - high-speed cameras; 3,4 - cross-correlation digital cameras; 5 - spotlight; 6 - personal compute; 7 - multichannel technological registrar; 8 - motorized pointing device (MPD) ; 9 - power supply unit MPD; 10 - aluminum rack; 11 - water tank; 12 - dosing devise; 13 - quartz tube; 14 - catcher; 15 - hollow tube with flammable liquid; 16 - digital multimeter; 17 synchronizer of computer, cross-correlation cameras and laser; 18 - double-pulse laser; 19 - laser line generator ; 20 - exhaust hood; 21-water supply tubing; 22 - thermocouples 23 - remote on/off switch; 24 - diffusion screen with a light guide.

Cycle experiments included two series. Recorded videos sprayed water entering the cylindrical channel with a flame (combustion temperature in the experiments was between $500 \mathrm{~K}$ to $2000 \mathrm{~K}$ ) in the first series of experiments. Recorded images of droplets after passing the flame of the combustion zone in the second series of. Movement sprayed working fluid in the high temperature gas medium recorded video cameras 1 and 2 (the picture format $-1024 \times 1024$ pixels, the frame rate - to $6 \cdot 10^{5}$ per second), and the cross-correlation cameras 3 and 4 (the figure format $-2048 \times 2048$ pixels, minimal delay between two sequence figures - not more than $5 \mathrm{~ms}$ ). The cameras directly connected with the operation of the laser 18 . The height of the installation of cross-correlation cameras 3,4 and the laser 18 chosen according to the methods for determining the speed and size of fine particles sputtered.

Installation height of the camera and laser chosen so that the optical axis of the camera and the light "pulse" lasers intersect at an angle of 90 degrees for methods PIV and IPI. Front the camera sets the diffusion screen 24 connected to the laser light guide 18 (installation height of the laser in this case is not important) for SP methodology in [6]. All captured video passed on to a workstation, where performed their processing and determination of the required parameters with the help of specialized software "Tema Automotive" [7].

The methodology defined depending on the parameter to be determined. IPI and SP methods used to measure droplet size. IPI method based on recording the instantaneous distribution of the diameters of droplets or bubbles in the flat section of the flow. The size of a spherical particle calculated from the interference pattern that observed in the defocused image of the particle. The SP method based on the recording of the shadow pictures of the object, which has a refractive index not like his environment. Digital analysis of the shadow image to determine the position and the border of the object [8].

Errors in determining droplet size reached $0.001 \mathrm{~mm}$. Random error of measurement of the mass of drops no more than $1 \%$. The error in determining the velocity of droplets is not more than $2 \%$. 


\section{Results and discussion}

Figure 2 shows the dependence of the parameter $\Delta R$ (the proportion of evaporated droplets) from the initial temperature of the liquid. We established that increasing the initial temperature of the water even at $10 \mathrm{~K}$, can increase the rate of evaporation of the droplets in twice.

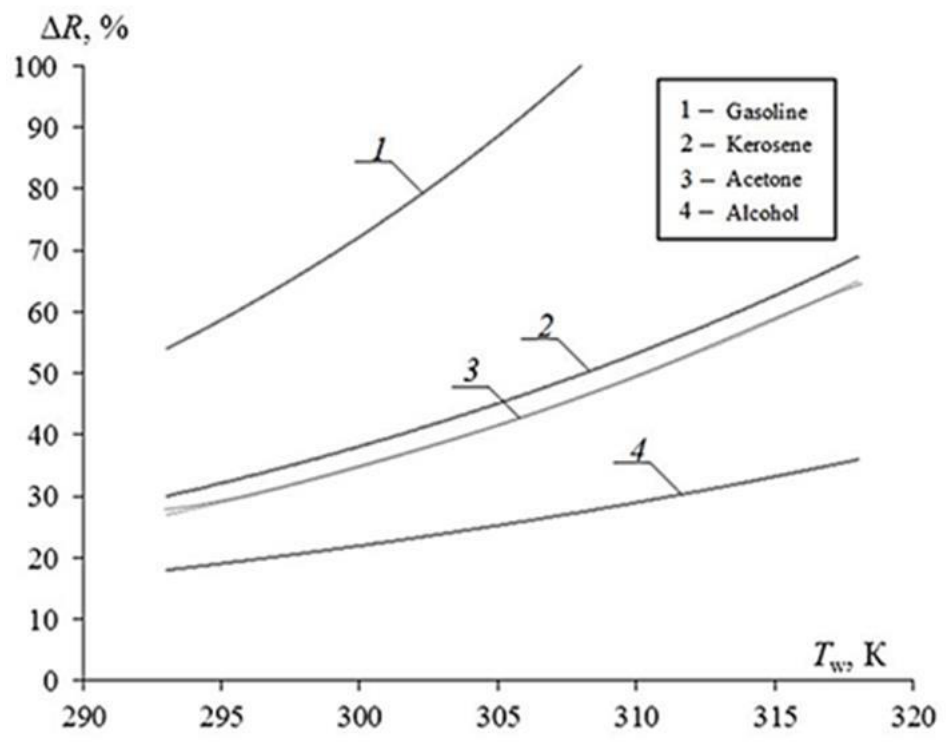

Figure 2. The experimental dependence of the parameter $\Delta R$ initial fluid temperature $T_{\mathrm{w}}$.

The type flammable liquid also influences the rate of evaporation of water droplets. Therefore, in the combustion products of gasoline droplets evaporate much more intense than the burning of industrial alcohol. Gasoline has a high temperature combustion products $-1906 \mathrm{~K}$. Acetone and kerosene burn with less intensity, their temperature no more than $1744 \mathrm{~K}$ and $1738 \mathrm{~K}$. The combustion products of alcohol have the smallest temperature $-1150 \mathrm{~K}$.

Figure 3 shows the effect of the initial size of the dispersed water droplets on the process of evaporation in the high temperature environment.

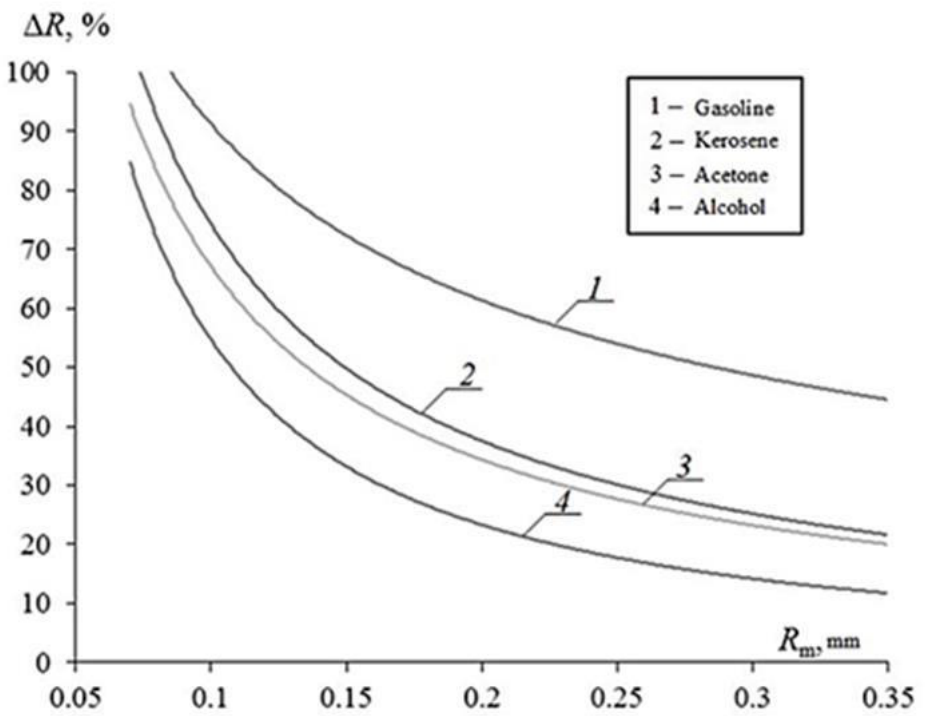

Figure 3. Experimental parameters $\Delta R$ depending on the initial droplet size $R_{\mathrm{m}}$.

Analysis the dependence showed that the small droplet better evaporate. The water by reducing evaporation time, increase the heat transfer coefficient and absorption capacity with decreasing droplet size is more suitable to extinguish than large drops of 


\section{EPJ Web of Conferences}

water. However, there is a limit for drops. This conclusion obtained in the study process braking and ash drops high temperature gases.

The study found that the processes of braking and turn of the dispersed water droplets influence the initial size $R_{\mathrm{m}}$ drops. Droplets with a diameter of 0.1-0.2 mm could not overcome the counter-resistance high-temperature environment. The resulting drops are stopped and turned. Drops optimum diameter reached the center of ignition. In addition, it should note that the degree of braking influences temperature counter gas stream. The droplets evaporate rapidly, slowing its movement. The more gas medium temperature, the more quickly drops braked.

\section{Conclusion}

1. The results showed the intensity of the process of evaporation of water spray greatly influenced by the initial size and the temperature of the water mist.

2. We have established that the products of combustion of gasoline droplets evaporate much more intense than in the combustion products of alcohol. This is due to the difference in temperature of the combustion products of the substances.

3. Investigated the process of carryover and turn drops having a smaller size, high temperature gases. Then it was found that the most effective use of plants with water mist necessary spatial differentiation droplet size (relatively large drops should be placed around the perimeter of the flow of smaller droplets).

The investigations were supported by the Russian Science Foundation (project No. 14-39-00003).

\section{References}

1. R. Wighus, Proceeding of the International Water Mist Conference (Vienna, 2001).

2. M. Na, J. Fire Sci., 30, 138 (2012)

3. Yuji Kudo, Yudai Kudo, Hiroyuki Torikai, Akihiko Ito, Fire Saf. J. 45, 2 (2010)

4. Xiangyang Zhou, Hong-Zeng Yu, Fire Saf. J., 46 (3), 140 (2011)

5. Di Wu, D. Guillemin, A. W. Marshall, Fire Saf. J., 42 (4), 283 (2007)

6. Xi-Shi Wang, Xiang-Di Zhao, Yi Zhang, Xin Cai, Rui Gu and Hong-Li Xu. J. Fire Sci., 27, 545 (2009)

7. R.S. Volkov, G.V. Kuznetsov, P.A. Strizhak, Thermophysics and Aeromechanics, 21 (2), 255 (2014)

8. L. Zlatanovic, J. Vreeburg, J. Peter van der Hoek, I. Poznakovs, Fire Saf. J., 68, 30 (2014)

9. J.V. Simo Tala, S. Russeil, D. Bougeard, J.-L. Harion, /Therm. and Fluid Sci., 50, 45 (2013) 